\title{
Georges Perec artisan de la langue, sous la direction de Véronique Montémont et Christelle Reggiani
}

\section{Laura Brignoli}

\section{(2) OpenEdition}

1 Journals

\section{Edizione digitale}

URL: http://journals.openedition.org/studifrancesi/2272

DOI: 10.4000/studifrancesi.2272

ISSN: 2421-5856

\section{Editore}

Rosenberg \& Sellier

\section{Edizione cartacea}

Data di pubblicazione: 1 aprile 2014

Paginazione: 182-183

ISSN: 0039-2944

\section{Notizia bibliografica digitale}

Laura Brignoli, « Georges Perec artisan de la langue, sous la direction de Véronique Montémont et Christelle Reggiani », Studi Francesi [Online], 172 (LVIII | I) | 2014, online dal 01 avril 2014, consultato il 18 septembre 2020. URL : http://journals.openedition.org/studifrancesi/2272 ; DOI : https://doi.org/ 10.4000/studifrancesi.2272

Questo documento è stato generato automaticamente il 18 settembre 2020.

\section{(c)}

Studi Francesi è distribuita con Licenza Creative Commons Attribuzione - Non commerciale - Non opere derivate 4.0 Internazionale. 


\title{
Georges Perec artisan de la langue, sous la direction de Véronique Montémont et Christelle Reggiani
}

\author{
Laura Brignoli
}

\section{NOTIZIA}

Georges Perec artisan de la langue, sous la direction de Véronique MONTÉMONT et Christelle REGGIANI, Lyon, Presses Universitaires de Lyon, 2012, pp. 195.

1 Il volume riunisce quindici articoli d'interesse specificamente linguistico, motivato dalla scarsità di studi simili sull'insieme dell'opera di Georges Perec come dal preteso disinteresse dell'autore per questo aspetto della scrittura. Ponendosi in antitesi rispetto alla vulgata che attribuirebbe a Perec una sorta di écriture blanche, gli studiosi forniscono interpretazioni stilistiche dei fatti linguistici. Va detto che nessuno di essi è nuovo all'approccio, come dimostra la bibliografia che chiude il volume. Diviso in tre sezioni («La Langue/les langues», «Peut-on parler d'un style perecquien?», «L'Art de la liste»), esso si presenta dunque come un valido strumento di approfondimento su un aspetto che sta stimolando la critica.

2 L'analisi quantitativa effettuata da Valérie MONTÉMONT (Du lexique perecquien, pp. 19-30) grazie a Frantext ha messo in luce aspetti interessantissimi della scrittura perecchiana mostrando non solo quali siano i campi semantici più sfruttati ma anche come questi siano utili a comprovare l'effettiva fondatezza linguistica di certi fatti, quali la mancanza di introspezione sostituita dall'accumulazione quasi maniacale degli oggetti. L'eccesso lessicale va a colmare l'assenza mostrando che tutta l'opera si cristallizza intorno a quella mancanza originaria che è il vero motore della scrittura. Jacques DÜRRENMATT (Que dit la ponctuation de Perec, pp. 31-41) riflette sul senso della punteggiatura, offrendo un'analisi minuziosa degli usi particolari, senza da ciò dedurre alcuna interpretazione generale. L'articolo di Isabelle DANGY (Les Verbes mode d'emploi: la 
conjugaison dans "La Vie mode d'emploi", pp. 43-53), enucleando grazie ai tempi verbali le parti descrittive e le parti narrative, evidenzia la presenza di uno degli aspetti davvero più ignorati della produzione di Perec: il romanzesco, la portata affabulatoria dei suoi romanzi, che lui sognava fossero non letti, ma divorati "sul letto a pancia in giù". Dopo aver mostrato cosa implica la soppressione della $e$ in termini di riduzione effettiva delle parole utilizzabili, Marc PARAYRE (Grammaire du lipogramme: "La Disparition", pp. 55-66) prende in considerazione i mezzi utilizzati da Perec per porvi rimedio, e il voluto effetto di banalizzazione ottenuto dal ritorno di certe scelte obbligate come l'anteposizione dell'aggettivo. Isabelle PARNOT (Faufilage à l'anglaise d'un sujet sans langue, pp. 67-76) mostra le valenze stilistiche degli anglicismi nelle Revenentes. Contrariamente a quanto avviene nella Disparition, qui vengono segnalati gli aspetti spiccatamente stilistici dell'uso degli anglicismi. Grazie alla sua pratica di traduttore, Hermes SALCEDA (Transposer la variation du "Petit vélo" en espagnol, pp. 77-87) mostra come certi testi costringano ad allontanarsi dalle abitudini codificate della traduzione per andare a esplorare tutte le risorse della lingua d'arrivo al fine di riprodurre quelle stesse variazioni lessicali e sintattiche.

3 Nonostante l'importanza del vuoto, dell'assenza, della mancanza come fondamento dell'opera di Perec, il "bianco" della sua scrittura (Maryline HЕCK, L"écriture blanche" de Georges Perec, pp. 91-102), che è una caratteristica stilistica e non solo un tema, ha anche il valore positivo di punto di partenza capace di favorire l'emergenza del soggetto. Dal bianco parte la scrittura che è affermazione di vita. Julien LONGHI (Un «autre versant $d u$ langage»: la relation fond/forme et figure/fond dans deux ceuvres de Perec, pp. 103-112) si avvale di alcuni principi mutuati a livello psicologico dalla Gestalt e a livello filosofico dalla semiotica che sostengono l'imprescindibilità di una percezione globale come fondo sul quale viene poi sviluppata la forma significante. E ne fornisce due esempi reinterpretando La Disparition e W. Lo sguardo di sbieco (Yvonne GOGA, "Douze regards obliques", un discours d'indirection, pp. 113-119) è una delle caratteristiche perecchiane che, in Penser/Classer, insegna al pensiero come assumere una direzione diversa dalle imposizioni della moda. Secondo Marie BONNOT (Écriture de rêve et jeux de mots dans "La Boutique obscure", pp. 121-130) la trascrizione dei sogni si accompagna a un lavoro consapevole sul linguaggio per fare in modo che il gioco di parole funga da mezzo fàtico e si mostri così che il sogno non è necessariamente un mezzo per lavorare sull'inconscio, ma ciò che conduce all'ipercoscienza linguistica. Dominique MONCOND'HUY (Écrire l'ordinaire: le jeu de l'intime et du collectif dans "Espèces d'espaces", pp. 131-141) mostra come questo testo sia tutt'altro che una serie di annotazioni più $o$ meno occasionali: opera che risponde a una ricerca sociologica da un lato e a una pratica autobiografica dall'altro, in cui l'intimo si combina con il collettivo, la sua aderenza a un principio strutturale come quello della massima alla Rochefoucauld, oltre agli intertesti pascaliani, ne fanno emergere la profondità metafisica.

L'ultima sezione contiene quattro studi sulla pratica delle enumerazioni, indagata nel profondo da Florence DE CHALONGE (Stylistique de l'indifférence: "Un homme qui dort", pp. 145-153) che rintraccia i mezzi linguistici usati da Perec per ottenere l'effetto stilistico dell'indifferenza. La lista, classificazione delle cose prima della loro ineluttabile scomparsa (Catherine RANnoux, Les Effets de liste dans "Espèces d'espaces" et "Les Choses", pp. 155-163), è un motore per l'invenzione di Perec (Lucia MANEA, Pratique de la liste des lieux et effets de style perecquiens, pp. 175-184) e, come appare attraverso gli articoli giovanili dell'autore, le enumerazioni nelle Choses (Matthieu RÉMY, Essai de 
contextualisation des usages énumératifs dans "Les Choses", pp. 165-173) non sono solo testimonianza di un mondo, ma sguardo critico su di esso. 